\title{
Enquête
}

Archives de la revue Enquête

$4 \mid 1988$

Varia

\section{À propos de quelques travaux de Lazarsfeld et de son école}

Media, leadership et interaction : une sociologie des pouvoirs invisibles

Michel Grumbach et Nicolas Herpin

\section{CpenEdition}

\section{Journals}

Édition électronique

URL : http://journals.openedition.org/enquete/65

DOI : 10.4000 /enquete. 65

ISSN : 1953-809X

Éditeur :

Cercom, Éditions Parenthèses

Édition imprimée

Date de publication : 2 juin 1988

Référence électronique

Michel Grumbach et Nicolas Herpin, "À propos de quelques travaux de Lazarsfeld et de son école », Enquête [En ligne], 4 | 1988, mis en ligne le 27 juin 2013, consulté le 19 avril 2019. URL : http:// journals.openedition.org/enquete/65; DOI : 10.4000/enquete.65

Ce document a été généré automatiquement le 19 avril 2019 


\title{
À propos de quelques travaux de Lazarsfeld et de son école
}

Media, leadership et interaction : une sociologie des pouvoirs invisibles

\author{
Michel Grumbach et Nicolas Herpin
}

1 En France, aucune des enquêtes que Lazarsfeld et ses collaborateurs ont consacrées à la communication et aux relations interpersonnelles n'a été traduite : The People's Choice ${ }^{1}$; Voting $^{2}$; Personal Influence ${ }^{3}$ et Medical Innovation ${ }^{4}$. On a généralement préféré d'autres textes, historiques et, surtout, méthodologiques. Rive droite, on paraît apprécier la méthode; rive gauche, on déplore l'absence d'idées et/ou la soumission à la " demande sociale ». Paradoxalement, partisans et adversaires ont concouru à accréditer la même image pour ne diverger que sur la valeur à accorder à l'entreprise: méthodologue exemplaire ou praticien étroit, au fond c'est tout un.

2 Pourtant il est sans doute exact de dire que les travaux de Lazarsfeld et de ses collaborateurs ont radicalement transformé la sociologie des media. Encore cette formulation prête-t-elle à confusion en ce qu'elle postule un découpage identique des disciplines en France, où la place d'une telle spécialité est résiduelle ou introuvable, et aux États-Unis, où elle s'avère considérable. Aussi serait-il plus exact de dire : l'ensemble des domaines qui, là-bas, avaient rapport à la sociologie des communications de masse (politique, développement, loisirs, journalisme et media, publicité et marketing, etc.).

Ce qui donne leur unité à ces recherches c'est une interrogation sur les processus par lesquels une classe ou un groupe produit et maintient sa propre légalité, obtient de ses membres des opinions droites et des actes conformes. La réponse réside dans une théorie du leadership et de l'interaction comme instances de contrôle social « local ", c'est-à-dire propre à la classe ou au groupe. Mais il faut admettre que la question n'avait pas de sens dans les grandes théories du pouvoir parce que là rien n'y advient qui n'ait sa source dans un principe extérieur et supérieur : État, élite ou classe dominante. Partis à la recherche des preuves de l'efficacité des pouvoirs institutionnels, les lazarsfeldiens découvrent les petits pouvoirs invisibles auxquels est suspendue l'existence quotidienne. 


\section{L'expert et le compagnon}

4 Personal Influence consigne le parcours par lequel l'analyse a pris congé des deux fictions qui organisaient jusque-là cette sociologie. D'une part, le pouvoir d'imposition illimité dont l'optimisme diffusionniste, hérité du $\mathrm{XVIII}^{\mathrm{e}}$ siècle, crédite les productions des élites, toujours portées à croire en la validité et la nécessité universelles de leurs propres valeurs. Soit, illusion corporative et fonctionnelle de tout pouvoir symbolique. D'autre part, l'individualisme atomiste inhérent à la notion de "société de masse", variante modernisée de l'« anomie » et de la Gesellschaft. Soit, une somme d'individus, chacun homo mediaticus, sans rapport les uns avec les autres et recevant leur unité de l'extérieur, c'està-dire des divers pouvoirs institutionnels qui sont censés les dominer absolument.

Du domaine politique à celui de l'activité scientifique, le constat est général : les messages des «sources expertes", ceux que diffusent media ou agents institutionnels, font moins autorité que ceux des partenaires ordinaires de la vie de tous les jours. Les mots de la tribu l'emportent sur ceux qui viennent d'ailleurs. Les divers pouvoirs symboliques ne s'imposent que pour autant que s'interpose une garantie indigène.

6 En se donnant pour la première fois les moyens de mesurer réellement l'impact des messages médiatiques - le panel et le schème de l'étude de décision -, The People's Choice puis Voting vont en découvrir les limites. Le premier constat par son importance, c'est la faiblesse du nombre des changements d'intention de vote susceptibles de se produire dans le cours d'une campagne électorale, en dépit de l'avalanche médiatique qu'elle suscite. Le second, c'est que les transferts d'allégeance qui se produisent sont plus souvent dus aux contacts face-à-face avec l'entourage (familial, professionnel ou amical) qu'aux discours électoraux ou aux éditoriaux des commentateurs. Parce que le niveau d'intérêt politique, la précocité du choix et sa fermeté, le volume et la sélectivité de l'exposition vont de pair, les communications formelles n'atteignent que ceux qui sont les moins susceptibles d'être influencés. Le résultat est inattendu: ou bien les messages n'atteignent pas, ou bien c'est en pure perte. Au lieu des changements en masse que la campagne était supposée induire, les auteurs découvrent la stabilité de l'opinion politique et l'homogénéité idéologique des groupes primaires. Même les changements individuels apportent leur contribution à cette uniformité parce qu'ils consistent le plus souvent en un alignement des membres déviants sur le vote tenu pour socialement correct dans leur entourage. Le citoyen éclairé que postule la philosophie politique classique s'avère introuvable : exit l'homo politicus.

7 Mais ces constats sont-ils généralisables ? Ce qui prévaut dans les circonstances somme toute exceptionnelles d'une campagne électorale se retrouve-t-il en des domaines plus prosaïques de l'existence?

8 Personal Influence tente cette confrontation des pouvoirs respectifs de l'amateur et de l'expert à l'aide d'un échantillon exclusivement féminin cette fois. La mode, l'achat de produits alimentaires ou d'entretien et les choix cinématographiques fournissent le contenu de ce test. Le message publicitaire de la radio ou de la presse, le magazine spécialisé ou le vendeur l'emportent-ils sur les contacts personnels et le bouche à oreille?

9 À nouveau, il ressort de cette enquête, et dans tous les cas, que l'influence personnelle joue un rôle plus important et plus efficace que n'importe quel moyen de communication de masse. 

le conjoint ou le collègue, après tout pourquoi pas? L'on est d'autant plus prêt à l'admettre qu'en matière d'élections, de consommation, de mode ou de cinéma l'expertise reconnue est faible et diffuse, ou qu'il s'agit de domaines où chacun est supposé avoir droit à l'opinion. Aussi n'est-il pas tout à fait surprenant d'y découvrir de l'influence informelle. L'idée serait plausible mais de portée limitée. En revanche le modèle serait soumis à un test bien plus probant si l'on pouvait faire état de constats analogues en des activités qui a priori ne doivent rien à l'opinion.

11 C'est ce que Medical Innovation s'efforce de démontrer. Dans une profession où l'idéologie de la Science et l'engagement vis-à-vis du malade tendent à faire du progrès une norme officielle, et dominée par des autorités fortement reconnues, l'adoption d'un nouveau médicament suit grosso modo un processus comparable à la diffusion de quelque nouvelle pratique agricole, telle l'acclimatation de l'hybride de maïs, parmi les fermiers de l'Iowa ${ }^{5}$. Si la précocité d'adoption, la forme du processus de diffusion et l'ensemble avec lequel agissent les médecins dépendent à ce point du nombre de liens et de contacts qu'ils entretiennent avec leurs collègues, c'est que ceux-ci détiennent, au regard des décisions $\mathrm{du}$ praticien, particulièrement en situation d'incertitude et de risque, une fonction de légitimité et de réassurance plus décisive que celle dont jouit l'élite professionnelle au travers de ses articles dans la presse médicale. Ces scientifiques dépendent tout autant de leurs collègues et $\mathrm{du}$ bouche à oreille que le citoyen ordinaire en quête d'une interprétation de la conjoncture du marché électoral.

12 L'enquête réalisée par $\mathrm{H}$. Menzel auprès de scientifiques (chimistes, biochimistes et zoologistes) montre combien il est socialement irréaliste de réduire l'information à l'idée d'une recherche intentionnelle mettant en relation un chercheur individuel isolé et une source selon des procédures exclusivement rationnelles ${ }^{6}$. Seuls les échanges informels permettent, en effet, à des chercheurs de maîtriser une information dont ils ignorent la pertinence, voire jusqu'à l'existence, ou qui excède, en raison de son volume et de sa dispersion, leurs possibilités d'investigation individuelles. Grâce aux relations interpersonnelles ceux-ci sont en mesure et de disposer d'informations qu'ils ne cherchent pas et de se procurer une bonne part de celles qu'ils cherchent.

L'issue de la confrontation est claire : les messages médiatiques, aussi autorisés soient-ils, n'ont pas le pouvoir qu'on leur prêtait de façonner aussi directement et facilement leurs auditoires, citoyens ou médecins. Parce qu'une autre légitimité, diffuse celle-là, s'interpose entre les individus et les pouvoirs institutionnels : celle de la classe ou du groupe d'appartenance. À l'omnipotence du contrôle social à distance, les lazarsfeldiens opposent la force du contrôle social « rapproché », lesté de tout le poids des sanctions et des valeurs du groupe.

\section{Leadership et interaction, ou les handicaps de la distinction}

Ces diverses enquêtes ont découvert le pouvoir régulateur considérable qu'exercent les relations interpersonnelles au travers de la diffusion de l'information, de la légitimation des décisions et du soutien procuré. Là réside la limite à l'action formatrice des messages des autorités distantes, quel que soit le degré de reconnaissance dont elles bénéficient. 
15 Le propre de la problématique lazarsfeldienne tient au rôle central qu'elle fait jouer aux relations interpersonnelles en tant que processus de contrôle social «local », c'est-à-dire endogène sur les plans social et culturel. Les relations interpersonnelles constituent une instance locale d'ordre et de légitimité. Deux relations concourent à la production et au maintien de l'opinion «correcte »: l'une est de pouvoir, c'est le leadership d'opinion; l'autre est symétrique et plus fondamentale, c'est l'interaction. Les deux thèmes, introduits dès The People's Choice, seront successivement accentués.

La prise en considération de l'influence prend une double valeur critique. Par rapport aux courants d'inspiration "communautaire " (Gemeinschaft), il implique que soit prise en compte l'asymétrie dans les relations sociales. Mais, à l'endroit des théories de style « sociétaire » (Gesellschaft), c'est l'aspect informel et familier de la domination qui est mis en avant. Les lazarsfeldiens intègrent le pouvoir aux théories des relations interpersonnelles et/ou les relations interpersonnelles aux théories du pouvoir. En outre, parant au naturalisme ouvert ou latent de nombre de doctrines de la domination, à la recherche de "traits", ils soulignent que l'influence est une relation ni générale, ni permanente, ni intransitive, ni décontextualisable ${ }^{7}$.

17 Ces détenteurs de l'ordinaire au pouvoir que sont les leaders d'opinion ont pour caractéristique première de n'être guère différents de ceux qu'ils influencent. Situés parmi les proches - conjoints, parents, collègues ou amis -, faisant office de conseiller écouté, ils ne se distinguent de leurs partenaires que par la compétence spéciale dont ils sont crédités, une sociabilité plus importante et davantage de contacts avec le monde extérieur à leur cercle, en particulier à travers les media. Remise de soi, l'influence personnelle est une imposition «douce » et sollicitée qui se produit dans le cours des contacts quotidiens entre des personnes liées par l'interconnaissance et partageant, pour l'essentiel, des caractéristiques et des opinions semblables. La découverte du leadership d'opinion implique un réexamen des théories classiques du pouvoir.

On sait le rôle exorbitant qu'ont joué les phénomènes de mode comme prototype du changement social par diffusion «verticale» (Simmel; Veblen; Halbwachs, etc.). Le scénario en est bien connu : les classes privilégiées sont les premières à adopter un bien nouveau, qu'elles abandonnent ensuite pour un autre lorsqu'elles sont imitées par les classes moyennes puis populaires. Contre ce modèle "vertical ", exclusif et récurrent, d'une domination généralisée des classes supérieures ou des élites, point focal où se concentreraient pouvoir, légitimité et innovation et à partir duquel, par percolation, toute la société serait mise en forme, les lazarsfeldiens inventent, avec l'influence " horizontale ", un schème nouveau, à usage alternatif ou complémentaire, en découvrant des structures de pouvoir internes à chaque classe, strate ou groupe - le leadership d'opinion comme relation de domination rapprochée et familière. À l'inverse de ce qu'implique le "modèle du percolateur ${ }^{8}$ ", hiérarchie et distance sociales apparaissent désormais comme des limites tant à la communication qu'au pouvoir. Bref, ici comme chez Newton, la force d'attraction varie en relation inverse de la distance.

Un second processus, plus fondamental que le précédent, contribue à la structuration des pratiques ou des attitudes : l'interaction.

20 Aussi déterminées que soient les «prédispositions politiques », par exemple, c'est-à-dire la probabilité d'un certain vote étant données les caractéristiques sociodémographiques des individus, la position politique, l'argumentaire nécessaire pour y parvenir, le discours d'accompagnement et l'acte de voter n'en constituent pas moins des réalités qui doivent 
être produites comme telles, portées « au niveau de la visibilité et de l'expression ${ }^{9}$ ». Sauf à admettre l'«automatisme anonyme » et le psychologisme qu'implique toute analyse prétendant déduire directement les comportements individuels des seules conditions objectives (i.e. de la structure sociale), «il est évident que la plus-value ne s'investit pas toute seule » comme l'écrit J. Schumpeter ${ }^{10}$.

Le rôle central que la problématique lazarsfeldienne fait jouer à l'« expression", sa production et son contrôle, ainsi qu'à l'échange verbal, n'est pas la moindre de ses originalités. C'est par et dans l'activité conversationnelle quotidienne que les acteurs inventent, constituent ou adaptent l'équipement symbolique nécessaire à leur accomplissement de la vie sociale. En ce sens, les lazarsfeldiens ont posé l'idée générale d'une théorie de la conversation comme processus de production des significations et des actions socialement correctes, c'est-à-dire localement légitimes. Maïeutique, constitutif ou validant, le dialogue ordinaire entre égaux est une instance productive et dotée d'une efficacité propre. Le modèle de l'action par contact se substitue à celui de l'impetus, force motrice immanente au mobile. Interaction et conversation constituent un processus d'influence sans « influent » et fournissent, dans le langage des auteurs, le principe d'une « sociologie symétrique ${ }^{11}$ ».

Sans en faire précisément une propriété suffisant à définir le leader d'opinion, The People's Choice met l'accent, à plusieurs reprises, sur la dimension de la compétence verbale ( « articulate individual » est le leader) nécessaire à son rôle de pourvoyeur de significations. En outre, le même ouvrage relève l'existence, à côté du leadership d'opinion, d'un second processus de formation de l'opinion, qualifié d'« émergence " ou de "cristallisation", dont s'acquittent les interactions, en dehors de toute relation de domination ${ }^{12}$.

Mais c'est avec Voting que l'interaction est spécifiée en tant que conversation et que cette dernière est explicitement liée à la production et au contrôle des comportements politiques :

«Donc l'environnement social de l'électeur-type est politiquement homogène.

Comment en est-il ainsi ? Et comment le demeure-t-il ? Une réponse est : "à travers

la discussion politique"13.»

24 L'économie des discussions politiques fournit le principe organisateur de l'analyse : la stabilité ou le changement de l'intention de vote dépend d'abord de la nature des positions politiques des interlocuteurs. Par exemple, la probabilité sociale de rencontrer, dans l'environnement habituel, un soutien conversationnel est le principal facteur de stabilité de l'attitude politique. De même, l'adoption d'une nouvelle préférence est fonction de la participation à des discussions avec les partisans de cette position :

«Par le processus même du dialogue, les dispositions vagues des gens se cristallisent, pas à pas, en attitudes, actes ou votes déterminés ${ }^{14}$. »

Voting propose une théorie du contrôle social rapproché, essentiellement voué au maintien des loyautés de classe, dont le principe réside dans une sociologie des fonctions symboliques de la conversation.

Entre le vote final et les «prédispositions politiques » s'intercale, pour les confirmer ou les corriger, la médiation efficace des échanges verbaux. Jamais invoquées contre les conditions objectives, les relations interpersonnelles n'ont d'autre rôle, dans l'analyse lazarsfeldienne, que d'acheminer ou d'actualiser les effets de la structure sociale. Parfois au prix d'un arbitrage lorsque les appartenances conduisent à des loyautés contradictoires. Si elle n'a rien du « monstre froid » d'une macrosociologie rudimentaire, 
la sociologie de Lazarsfeld n'est pas d'avantage un psychologisme ou une théorie de l'intersubjectivité.

Un thème général domine cette sociologie: l'importance prioritaire accordée à la dynamique interne propre des classes sociales, à travers le commerce de leurs membres ou la contribution des «leaders naturels ». Le marxiste viennois est-il vraiment si loin derrière le sociologue américain?

\section{Des objets en quête de méthodes}

La problématique de l'influence interpersonnelle fait mauvais ménage avec les principes de la survey research, i.e. l'ensemble des instruments et des procédures de l'enquête par sondage. De fait, la première n'a pu être conquise que contre certaines des règles de la seconde. Et que les deux doivent autant à Lazarsfeld constitue une singularité qui explique sans doute bien des aspects de l'entreprise. Par exemple, si la redéfinition de l'objet de la sociologie des media a permis d'apercevoir quelques-unes des limites de l'enquête quantitative, en revanche, l'adhésion exclusive aux idéaux de la démonstration statistique, en portant à faire l'impasse sur le recueil des données jugées rebelles à l'observation standardisée et à la mesure, a systématiquement privé les auteurs d'une partie des preuves nécessaires. Jamais les lazarsfeldiens n'ont désespéré de pouvoir concilier intégralement l'étude des réseaux interpersonnels et des interactions avec les avantages qui tiennent à l'administration de la preuve dans l'enquête par sondage ${ }^{15}$.

Dans leur recherche permanente d'une méthodologie adéquate à leur projet théorique, les lazarsfeldiens n'ont cessé d'être confrontés aux canons de l'enquête par sondage qu'ils avaient eux-mêmes contribué à rationaliser et à imposer.

Le principal obstacle qu'oppose la philosophie de la survey research à la saisie d'un objet dont l'essence relationnelle fait toute la réalité, c'est l'individualisme atomiste qui guide la totalité de ses actes. Il inspire, en effet, tant les choix d'échantillonnage - l'échantillon aléatoire et représentatif d'individus - que la sélection des données bonnes à recueillir, et donc celle des variables pertinentes pour l'interprétation - les propriétés (structurales) « individuelles" plutôt que les relationnelles - ou encore la constitution des unités d'analyse - les classes d'individus plutôt que les classes de relations.

31 L'analyse de E. Katz, relative à The People's Choice, montrant comment le dispositif de cette enquête ne permettait en rien d'affirmer, comme le firent les auteurs, l'existence du leadership d'opinion comme relation liant leaders et non-leaders, a été souvent reprise. Voting, qui reconduit la méthodologie de la précédente étude (échantillon aléatoire et représentatif d'individus; panel et leaders auto-désignés), la modifie sur un point crucial et tente une nouvelle solution. Parce que l'on interroge systématiquement chacun sur les caractéristiques et les opinions des membres de son environnement (conjoint, parents, amis, collègues), de ses interlocuteurs ainsi que sur le sujet et la nature de ses conversations, il est possible de mettre les comportements des interviewés en relation avec ceux des individus de leurs différents cercles. Avec l'adoption de l'échantillon en "boule de neige ", qui procède de l'influenceur à l'influencé ou l'inverse, la démarche de Personal Influence permet à la fois d'identifier les leaders d'opinion sur la base d'une relation réelle et d'un jugement indépendant et de confronter les paires de partenaires liés par des transactions effectives. Enfin, si l'on considère Medical Innovation, le recensement de quelques groupes réels remplace les types d'échantillon précédemment 
utilisés; la détermination des leaders d'opinion repose sur la cartographie complète de la structure sociométrique de la communauté professionnelle; et l'analyse vise essentiellement à mettre en relief le rôle déterminant des variables d'«intégration sociale » et des relations, qu'il s'agisse de paires ou de « cliques », etc. ${ }^{16}$.

Chacune de ces innovations a entraîné peu ou prou leurs auteurs hors des chemins habituels de l'enquête par sondage sans pour autant leur garantir la solution des problèmes. La validité de la démarche de Voting fait question. Personal Influence bute sur les aléas de la "boule de neige » et de la confirmation du lien et du rôle déclarés qui rendent quasi imprévisibles tant le nombre de paires finalement disponibles que leur équi-répartition dans les diverses catégories sociodémographiques. Enfin, la méthodologie de Medical Innovation est rien moins que généralisable.

La règle tacite selon laquelle hors de la survey research, point de salut, constitue la seconde limite de ces travaux. Au fil de ses transformations successives, la problématique de l'influence interpersonnelle n'a cessé de proposer des objets qui sortent toujours plus du cadre de l'enquête statistique.

Medical Innovation devait avoir recours à la sociométrie. Voting, qui a désarticulé le schème du two-step flow bouclant, de façon commode et rassurante, le processus de diffusion en ses deux extrémités - avec des leaders d'opinion exclusivement voués aux media et des non-leaders uniquement en relation avec les leaders - propose, à qui n'admet que l'enquête quantitative, une tâche insurmontable avec sa notion de "circuits sans fin de relations de leadership qui traversent la collectivité ${ }^{17} »$. En outre, comment le questionnaire satisferait-il l'intérêt croissant porté au déroulement même des interactions et des conversations?

Mais le symptôme le plus visible des contraintes qu'impose le recours exclusif à l'enquête par sondage tient dans une lacune paradoxale et récurrente des données. À aucun moment, en effet, les auteurs n'ont pu documenter directement les formes concrètes de l'influence interpersonnelle en œuvre dans les interactions et les conversations. Toujours la reconstruction spéculative a dû pallier les carences de l'observation. L'analyse tourne court dès qu'elle doit rendre compte des modalités d'action de l'influence. Soit, dans Medical Innovation, l'exemple de l'analyse du processus d'adoption du nouveau médicament. L'examen des courbes révèle non seulement que les médecins «intégrés » l'ont prescrit plus tôt que leurs confrères « isolés ", mais encore que la forme même de la diffusion diffère : purement individuel pour les « isolés », le processus obéirait au modèle de la contagion dans le cas des «intégrés ». Cependant les données font défaut pour établir comment des praticiens en relation les uns avec les autres parviennent à une décision identique et quasi simultanée. Il n'y a rien pour documenter les modalités du phénomène de contagion, si central pour toute la démonstration :

«Les données de cette enquête ne permettent pas de décider laquelle de toutes ces transactions possibles (imaginées) est responsable de l'effet observé ${ }^{18}$. »

Le recours exclusif à l'enquête par sondage a constamment limité le déploiement de la problématique comme l'établissement des faits ${ }^{19}$. C'est bien en vain que Voting proposera l'idée d'une sorte de sociologie des conversations comme prolongement de la théorie de l'influence interpersonnelle.

La théorie des relations interpersonnelles a eu pour résultat le plus visible de dissiper la croyance en un pouvoir magique des media. Ou, à tout le moins, d'en relativiser les effets. 

figurantes " (selon les mots de Pascal) en affectant de ne pas voir que "derrière » les media se tiennent les Auteurs des messages qu'ils diffusent, c'est-à-dire les divers pouvoirs symboliques, et leur volonté de donner forme aux pratiques sociales. Car, dans la sociologie américaine des communications de masse, les media ne sont en fait qu'une «figure » de la mission civilisatrice des élites : Lumières, Éducation et Progrès. Or, c'est précisément cette variante moderne du «mythe de l'Éducation» que les enquêtes lazarsfeldiennes mettent à mal. À dire vrai, peut-être à leur corps défendant, tout au moins au début. L'ordre du monde s'est avéré moins malléable que prévu. Comme le remarque Medical Innovation, même les «bons" changements ne s'imposent pas d'euxmêmes. Le scénario s'inverse : la ruse se retrouve du côté du récepteur; à l'émetteur, la naïveté et l'illusion. De la politique à la science, en passant par la consommation et les loisirs, autant d'actes sur lesquels les proches de la tribu, experts-amateurs ou pas, exercent une judicature que les théories des pouvoirs plus visibles sont spontanément portées à oublier. Les pouvoirs les plus patents ou les plus légitimes peuvent être tenus en échec : tel est le constat répété. En ce sens, la sociologie des lazarsfeldiens est d'abord une théorie des limites du pouvoir des « sources expertes».

Mais de l'origine même de la désillusion devait jaillir ce qui passe à leurs yeux pour une large compensation: les démocraties n'en sont-elles pas plus robustes de dépendre davantage de la sagesse concertée de beaucoup que de la clairvoyance supposée de quelques-uns?

\section{NOTES}

1. P. Lazarsfeld, B. Berelson \& H. Gaudet, The People's Choice. How the Voter Makes up his Mind in a Presidential Campaign, New York, Columbia University Press, 1940, $2^{\mathrm{e}}$ éd.

2. B. Berelson, P. Lazarsfeld \& W. McPhee, Voting. A study of Opinion Formation in a Presidential Campaign, Chicago, The University ol Chicago Press, 1954.

3. E. Katz \& P. Lazarsfeld, Personal Influence. The Part Played by People in the Flow of Mass Communications, Glencoe (IL), Free Press, 1955.

4. J. Coleman, E. Katz \& H. Menzel, Medical Innovation. A Diffusion Study, The Bobbs-Merrill Cy, 1966.

5. Cf. E. Katz, "The social itinerary of technical change. Two studies of the diffusion of innovation ", in W. Schramm \& D. Roberts, eds, Process and Effects of Mass Communication, 1971, p. 761-797.

6. H. Menzel, "Planned and unplanned scientific communication", in Proceeding of the International Conference on Scientific Information, Washington DC, 1959.

7. Cf. R. Merton, «Patterns of influence. A study of interpersonal influence and communications behavior in a local community ", in P. Lazarsfeld \& F. Stanton (eds), Communications Research 1948-1949, New York NY, 1949.

8. Cf. C. Grignon, J.-C. Passeron, À propos des cultures populaires, ${ }^{\circ}$ sp. Enquête. Cahiers du CERCOM, $1,1985$.

9. Lazarsfeld et al., The People's Choice..., p. 75. 
10. Cf. J. Schumpeter, "Les classes sociales en milieu ethnique homogène ", in Impérialisme et classes sociales, Paris, Minuit, 1972, p. 174-175

11. Cf. Berelson et al., Voting..., p. 299-300.

12. Cf. Lazarsfeld et al., The People's Choice..., Préface à la seconde édition, p. XXIII-XXIV.

13. Berelson et al., Voting..., p. 101-102.

14. Ibid., p. 299-300 et cf. également p. 292-296.

15. E. Katz, "The two-step flow of communication. An up-to-date report on an hypothesis", Public Opinion Quarterly, 21, 1957; et «Diffusion: interpersonal influence», International Encyclopedia of the Social Sciences, 4, 1969.

16. Cf. Coleman et al., Medical Innovation..., p. 113-114.

17. Berelson et al., Voting..., p. 110.

18. Coleman et al., Medical Innovation..., p. 112. Cf. également p. 85 et 123-124.

19. Cf. J. Robinson, «Interpersonal influence in election campaigns. Two-step flow hypotheses », Public Opinion Quarterly, 40, 1976, p. 309. 